\title{
Asymmetry in Cortical and Subcortical Structures of the Brain in Children and Adolescents with Attention-Deficit/Hyperactivity Disorder
}

This article was published in the following Dove Press journal: Neuropsychiatric Disease and Treatment

\author{
Sijian Chen (1D)' \\ Lin Guan' \\ Jie Tang' \\ Fan $\mathrm{He}^{2,3}$ \\ Yi Zheng ${ }^{2,3}$
}

'Beijing Anding Hospital, Capital Medical University, Beijing, 100088, People's Republic of China; ${ }^{2}$ The National Clinical Research Center for Mental Disorders \& Beijing Key Laboratory of Mental Disorders, Beijing Anding Hospital, Capital Medical University, Beijing, 100088, People's Republic of China; ${ }^{3}$ Advanced Innovation Center for Human Brain Protection, Capital Medical University, Beijing, 100069, People's Republic of China
Correspondence: Yi Zheng The National Clinical Research Center for Mental Disorders \& Beijing Key Laboratory of Mental Disorders, Beijing Anding Hospital, Capital Medical University, Beijing, 100088, People's Republic of China

Email yizheng@ccmu.edu.cn
Background: Human cognitive and emotional functions are asymmetrical between the left and right hemispheres. In neuroimaging studies of attention-deficit/hyperactivity disorder (ADHD) patients, the absence of aberrant asymmetry might serve as a neuroanatomical marker of ADHD. However, few studies have estimated abnormalities in cortical and subcortical asymmetry in children and adolescents of different ADHD subtypes.

Methods: Data were from the results collected by the Peking University site in the "ADHD-200 sample" dataset, which comprised 31 eligible ADHD (20 inattentive ADHD (ADHD-I), 11 combined ADHD (ADHD-C)) and 31 matched typically developing (TD) individuals. The Asymmetry Indexes (AIs) in cortical thickness, cortical gray-matter volume and subcortical nucleus (SN) volume were calculated based on an automated surface-based approach. The differences in cortical thickness, cortical gray-matter volume, and SN volume AIs were evaluated among groups. We also analyzed the correlation between AIs and the severity of ADHD symptoms.

Results: Compared with the TD group, SN asymmetry in ADHD group did not reveal significant differences. Altered cortical asymmetry of different subtypes in ADHD groups was located in the orbitofrontal and anterior cingulate circuits, including the medial orbitofrontal, paracentral, pars triangularis, caudal anterior cingulate, isthmus cingulate, and superior frontal regions. In the comparisons, cortical gray-matter volume AIs were significantly different in the caudal anterior cingulate, isthmus cingulate, and superior frontal regions between ADHD-I and ADHD-C groups. There were significant correlations between the severity of ADHD symptoms and asymmetric measurements in medial orbitofrontal, paracentral and isthmus cingulate regions.

Conclusion: These findings provide further evidence for the altered cortical morphological asymmetry in children and adolescents with ADHD, and these differences are associated (at least in part) with the severity of ADHD symptoms. Brain asymmetry could be an appropriate precursor of morphological alterations in neurodevelopmental disorders.

Keywords: attention-deficit/hyperactivity disorder, child and adolescent, cortical and subcortical, asymmetry

\section{Introduction}

Attention-deficit/hyperactivity disorder (ADHD) is a neurodevelopmental disorder. It can affect the health and social function of children and adolescents to a large extent. The core deficits of ADHD are associated with controlled processes and executive functions, and executive deficits might be due (at least in part) to impairment in automatic processing. ${ }^{1,2}$ According to the Diagnostic and 
Statistical Manual of Mental Disorders, Fourth Edition, ADHD can be divided into three subtypes on the basis of the predominant symptomatology: the inattentive (ADHDI), the hyperactive/impulsive (ADHD-HI) or the combined ADHD (ADHD-C) subtypes. ${ }^{3,4}$ The worldwide prevalence of ADHD is about $5.0-7.1 \%,{ }^{4}$ and the prevalence of ADHD-I is the highest, followed by ADHD-C. ${ }^{5}$ The course of ADHD is usually chronic. The lifelong prevalence of ADHD can reach up to $15 \%,{ }^{6}$ which is closely associated with a high prevalence of recurrence and low compliance of children and adolescents with ADHD. Such a high lifelong prevalence of ADHD imposes a heavy burden upon society. Therefore, exploring further the pathogenesis of this neurodevelopmental disorder is becoming increasingly important.

Brain hemispheres have asymmetric characteristics in terms of structure and function. ${ }^{7}$ The dominant hemisphere for verbal cognitive function is the left hemisphere. The dominant hemisphere for spatial cognitive function is the right hemisphere. The most widely identified structurally asymmetric regions are the right-lateralization of the frontal lobe and left-lateralization of the occipital lobe. ${ }^{8,9}$ Studies have shown that changes in the asymmetry of the brain are associated with the sex, age, handedness, and neuropsychiatric disorders of individuals (eg, schizophrenia, ${ }^{10}$ major depressive disorder, ${ }^{11}$ and Alzheimer's disease). ${ }^{12}$ The absence of asymmetry in healthy people might serve as a neuroanatomical marker of neurodevelopmental disorders (especially ADHD). ${ }^{10,13}$

Numerous studies on the anatomic and functional differences in the brains of people suffering from ADHD have been carried out. A meta-analysis of the studies based on voxel-based morphometry reported a reduction in the gray-matter volume in the caudate and putamen of ADHD patients. ${ }^{14}$ A recent cross-sectional study explored if ADHD patients had smaller gray-matter volume in the amygdala, caudate, hippocampus, and putamen. ${ }^{15}$ Some functional magnetic resonance imaging (fMRI) studies have demonstrated that patients with ADHD have structural and functional abnormalities in cortico-striatal circuits. ${ }^{16,17}$ Abnormalities in these brain regions damage the cognitive functions of individuals, such as the abilities of attention, automatic processing, and executive monitoring. ${ }^{2,18}$ Silk and colleagues, using diffusion tensor imaging, showed that an ADHD group had anomalous hemispheric asymmetry in the fiber tracts of the frontostriatal system. ADHD patients did not have the rightlateralization of tract connections between the caudate and prefrontal cortex observed in typically developing (TD) controls. ${ }^{19}$ Therefore, the subcortical nucleus (SN) might play a unique part as a precursor of more distinct asymmetry in the human brain. Studies have mostly analyzed alterations in the gray-matter volume from a voxelbased perspective, ${ }^{4,14,20-22}$ which may underestimate subtle differences in cortical structure. The cortical graymatter volume is affected by several factors, such as surface area, thickness, and folding of the cortex. In contrast, cortical thickness (another commonly used measure of the cortex) could be more sensitive to disorder-related structural differences in the human brain. ${ }^{23}$ However, only one surface-based analysis has investigated the hemispheric asymmetry in young patients with ADHD. ${ }^{24}$ Moreover, no one has estimated whether and how different ADHD subtypes could affect the hemispheric asymmetry of cortical and subcortical structures of the brain.

Our study had two main aims. The first aim was to investigate the subcortical structural asymmetry as a potential subcortical precursor in children and adolescents with ADHD. Second, we assessed whether ADHD subtypes might influence the asymmetry of cortical and subcortical structures, and the association between these cortical and subcortical asymmetric alterations and symptoms in patients with ADHD.

\section{Materials and Methods \\ Participants}

The data of our study were from the "The ADHD-200 sample" dataset ${ }^{25}$ in the "1000 Functional Connections Project" project group. All data in the present study were from the results collected by the Peking University (Beijing, China) site, which included the brain imaging of children. We did not choose multi-site data so as to avoid the adverse effects of the collection equipment and environment of different sites on the results. In the database of the Peking University site, patients were recruited from the Outpatients Department of the Institute of Mental Health of Peking University. TD individuals were children and adolescents recruited from nearby schools who did not have a current or previous psychiatric diagnosis or known neurological disorder. All participants in our study were right-handed with an intelligence quotient (IQ) score $>80$. Patients with neurological disorders, neurodevelopmental disorders, schizophrenia, affective disorders, oppositional defiant disorders, and learning disorders were excluded. The database recorded only whether participants had 
medications but lacked detailed information on how long ago or whether ADHD participants had taken them recently. Hence, we did not exclude ADHD participants who were taking medications (seven cases with ADHD-I and five ADHD-C cases were not medication-naïve). All data from the Peking University site were approved by the Research Ethics Review Board of the Institute of Mental Health within Peking University. One parent of each participant and all children provided written informed consent before participating. The guidelines outlined in the Declaration of Helsinki 1964 and its later amendments were followed in the present study. All structural magnetic resonance imaging (sMRI) data and demographic data were complete and usable. Finally, 31 eligible samples of ADHD data (20 ADHD-I and 11 ADHD-C) were obtained, and data samples from 31 matched (age, sex, and IQ score) TD individuals were collected.

All participants were interviewed with the Schedule of Affective Disorders and Schizophrenia for School-Age Children-Present and Lifetime Version (K-SADS-PL) ${ }^{26}$ and full-scale Wechsler Intelligence Scale for Chinese ChildrenRevised (WISCC-R). ${ }^{27}$ The ADHD Rating Scale (ADHDRS) $I^{28}$ was employed to measure ADHD symptoms.

\section{MRI Analysis}

Before further analyses of brain images, we first confirmed that all sMRI scans downloaded from the database were unaffected by head movement or other artifacts to ensure the image quality. sMRI scans were processed automatically with the FreeSurfer 6.0 (http://surfer.nmr.mgh.harvard.edu) recon-all processing pipeline for whole-brain cortical morphological characteristics. After that, the processing results of each participant were checked and corrected manually. The SN includes the thalamus, caudate, putamen, globus pallidus, hippocampus, amygdala, and nucleus accumbens.

\section{Statistical Analysis}

Data were analyzed using SPSS 20.0 (IBM, Armonk, NY, USA). The two-sample $t$-test was employed for the parameters of two independent groups with a normal distribution (eg, age, IQ score, ADHD-RS IV score). The Mann-Whitney U-test was used for data with a non-normal distribution. The one-way ANOVA test was undertaken for the parameters of multiple groups that had a normal distribution. The Kruskal-Wallis Htest was employed for the parameters of multiple independent groups with a non-normal distribution. The chi-square test was used to compare sex distribution. The difference between the left and right hemispheres of each cortical and subcortical region was represented by the Asymmetry Index (AI):

$$
\mathrm{AI}=(\mathrm{L}-\mathrm{R} / \mathrm{L}+\mathrm{R}) \times 100
$$

where $\mathrm{L}$ is the value for the left hemisphere, $\mathrm{R}$ is the value for the right hemisphere, and the AI ranges from -100 (complete rightward asymmetry) to +100 (complete leftward asymmetry). The Kruskal-Wallis H-test was employed to compare the differences in the AIs for the volume of the $\mathrm{SN}$ between children and adolescents with different ADHD subtypes and that in TD controls. Simultaneously, according to the Desikan-Killiany template, each hemisphere of the cortical regions was divided into 34 regions. The Mann-Whitney and Kruskal-Wallis tests were used to evaluate the differences in the average cortical thickness AI and gray volume AI of each region. Finally, the correlation between the AIs and clinical characteristics of ADHD patients was analyzed.

\section{Results}

\section{Demographic and Clinical Characteristics}

Data for demographic characteristics and clinical characteristics for ADHD and TD groups are shown in Table 1a. The results of the two-sample $t$-test and chi-square test showed that there were no significant differences in age, sex, or IQ score between the two groups $(P>0.05)$. The severity of ADHD symptoms (subscale scores of ADHDRS IV) was significantly different between the two groups $(P<0.05)$. The subscale scores were higher in ADHD patients compared with those in TD participants.

The three groups (ADHD-I, ADHD-C, and TD) were matched in terms of age, sex, and IQ score $(P>0.05)$. One-way ANOVA was used to analyze the ADHD-RS IV scores of ADHD-I, ADHD-C, and TD groups. In the subscales of ADHD-RS IV, there were significant differences among the three groups $(P<0.05)$, and the subscale scores of the two ADHD-subtype groups were significantly higher than those of the TD group (Table 1b).

\section{Comparison of the Asymmetry of the SN Between ADHD and TD Groups}

The subcortical volume in the caudate, hippocampus, and accumbens was more leftward in the ADHD group compared with that in the TD group, and more rightward in the putamen. However, significant differences were not detected in the AIs for the SN volume between ADHD and TD groups $(P>0.05)$ (Table 2$)$. 
Table I Demographic and Clinical Characteristics

\begin{tabular}{|c|c|c|c|c|c|}
\hline $\begin{array}{l}\text { (a) } \\
\text { Characteristic }\end{array}$ & \multicolumn{2}{|l|}{$\begin{array}{l}\text { ADHD } \\
n=31\end{array}$} & $\begin{array}{l}\text { TD } \\
n=31\end{array}$ & $t \mid \chi^{2}$ & $\mathbf{P}$ \\
\hline Age & \multicolumn{2}{|l|}{ II.67 (I.82) } & II.76 (I.82) & 0.201 & $0.84 I$ \\
\hline Sex (boy/girl) & \multicolumn{2}{|l|}{$29 / 2$} & $24 / 7$ & 2.080 & 0.149 \\
\hline IQ & \multicolumn{2}{|l|}{$106.00(\mid 4.29)$} & $109.35(15.32)$ & 0.892 & 0.376 \\
\hline \multicolumn{6}{|l|}{ ADHD-RS IV } \\
\hline Inattention & \multicolumn{2}{|l|}{$28.29(3.24)$} & $15.35(4.4 \mid)$ & -13.168 & $0.000 *$ \\
\hline Hyperactivity/Impulsivity & \multicolumn{2}{|l|}{$21.61(6.01)$} & I3.307 (3.96) & -6.610 & $0.000 *$ \\
\hline (b) & ADHD-I & ADHD-C & TD & $F / \chi^{2}$ & $P$ \\
\hline Characteristic & $\mathbf{n}=\mathbf{2 0}$ & $n=I I$ & $n=31$ & & \\
\hline Age & $12.00(|.7|)$ & II.07 (1.93) & $1 \mathrm{I} .76(\mathrm{I} .82)$ & 0.976 & 0.383 \\
\hline Sex (boy/girl) & $18 / 2$ & $11 / 0$ & $24 / 7$ & 3.205 & 0.187 \\
\hline IQ & $103.20(13.78)$ & $111.09(14.40)$ & $109.35(15.32)$ & 1.430 & 0.248 \\
\hline \multicolumn{6}{|l|}{ ADHD-RS IV } \\
\hline Inattention & $27.85(2.32)$ & $29.09(4.48)$ & I5.35 (4.4I) & 86.664 & $0.000 *$ \\
\hline Hyperactivity/lmpulsivity & $18.45(4.73)$ & $27.36(3.11)$ & I3.307 (3.96) & 50.483 & $0.000 *$ \\
\hline
\end{tabular}

Notes: Continuous variables were expressed as the mean (standard deviation). *Significant at $P \leq 0.05$.

Abbreviations: ADHD, attention-deficit/hyperactivity disorder; TD, typically developing; ADHD-I, inattentive attention-deficit/hyperactivity disorder; ADHD-C, combined attention-deficit/hyperactivity disorder; IQ, intelligence quotient; ADHD-RS IV, ADHD Rating Scale IV.

\section{Comparison of the Cortical Regions and SN Asymmetry Between the Two Subtypes in ADHD and TD Groups}

The average cortical thickness AIs of 34 pairs of cortical regions extracted from the Desikan-Kiliany Atlas were compared between the two subtypes in ADHD and TD groups. There were significant differences in the cortical thickness AIs of the medial orbitofrontal, paracentral, and pars triangularis among the three groups $(P<0.05)$ (Table $3)$. In the pairwise comparisons, there was a significant

Table 2 The SN Volume Als Between ADHD and TD Groups

\begin{tabular}{|c|c|c|c|c|}
\hline \multirow{2}{*}{$\begin{array}{l}\text { Region } \\
\mathrm{SN}\left(\mathrm{mm}^{3}\right)\end{array}$} & \multicolumn{2}{|l|}{ Al } & \multirow[t]{2}{*}{$Z$} & \multirow[t]{2}{*}{$P$} \\
\hline & ADHD & TD & & \\
\hline Thalamus & $-1.40(1.74)$ & $-1.86(0.34)$ & 0.824 & 0.410 \\
\hline Caudate & $-2.00(2.30)$ & $-1.52(0.47)$ & -0.993 & 0.321 \\
\hline Putamen & $1.78(2.19)$ & $1.68(0.29)$ & 0.303 & 0.762 \\
\hline Pallidum & $4.66(2.99)$ & $5.12(0.62)$ & -0.246 & 0.805 \\
\hline Hippocampus & $-1.94(2.78)$ & $-0.96(0.45)$ & -1.232 & 0.218 \\
\hline Amygdala & $0.28(4.45)$ & $\mathrm{I} .09(0.8 \mathrm{I})$ & -0.697 & 0.486 \\
\hline Accumbens & $-3.76(5.32)$ & $-2.70(1.08)$ & $-0.64 I$ & 0.522 \\
\hline
\end{tabular}

Note: Continuous variables were expressed as mean (SEM).

Abbreviations: SN, subcortical nucleus; Al, asymmetry index; ADHD, attentiondeficit/hyperactivity disorder; TD, typically developing. difference between ADHD-I and TD groups in the cortical thickness AI of the medial orbitofrontal (adjusted $P=$ 0.003). Moreover, the cortical thickness AI of the paracentral was significantly different between ADHD-C and TD groups (adjusted $P=0.029$ ) (Table 4). However, the cortical-thickness asymmetry of the pars triangularis showed no significant difference in the pairwise comparisons. There were no significant differences in the corticalthickness asymmetry of these three regions between the two subtypes in ADHD (adjusted $P>0.05$ ).

Results for comparisons of the cortical gray-matter volume and SN volume AIs between the two subtypes in ADHD and TD groups are summarized in Table 5. The cortical gray-matter volume AIs of the caudal anterior cingulate, isthmus cingulate, and superior frontal were significantly different among the three groups $(P<0.05)$. The $\mathrm{SN}$ volume AIs did not reveal significant changes between the two subtypes in ADHD and TD groups $(P>0.05)$. In the pairwise comparisons, there were significant differences in the cortical gray-matter volume AIs of the caudal anterior cingulate, isthmus cingulate, and superior frontal regions between ADHD-C and ADHD-I groups (adjusted $P=0.050,0.036$, and 0.045 , respectively). For the comparison between ADHD-C and TD groups, the cortical gray-matter volume 
Table 3 The Cortical Thickness Als Between the Two Subtypes in ADHD and TD Groups

\begin{tabular}{|c|c|c|c|c|c|}
\hline \multirow[t]{2}{*}{ Region } & \multicolumn{3}{|l|}{ Al } & \multirow[t]{2}{*}{$\chi^{2}$} & \multirow[t]{2}{*}{$\mathbf{P}$} \\
\hline & ADHD & & TD & & \\
\hline Cortical regions (mm) & ADHD-I & ADHD-C & & & \\
\hline Bankssts & $-2.77(0.68)$ & $-0.79(0.98)$ & $-2.12(0.52)$ & 2.862 & 0.239 \\
\hline Caudal anterior cingulate & $3.74(1.05)$ & $3.89(1.58)$ & $1.80(0.79)$ & 2.125 & 0.346 \\
\hline Caudal middle frontal & $\mathrm{I} .4 \mathrm{I}(0.47)$ & $-0.42(0.58)$ & $0.89(0.39)$ & 5.623 & 0.060 \\
\hline Cuneus & $-0.18(0.65)$ & $-1.20(0.90)$ & $-1.02(0.54)$ & 1.634 & 0.442 \\
\hline Entorhinal & $-3.37(1.43)$ & $-1.25(0.95)$ & $-2.02(0.88)$ & 2.272 & 0.321 \\
\hline Fusiform & $-0.62(0.35)$ & $-0.61(0.36)$ & $0.04(0.37)$ & 1.186 & 0.553 \\
\hline Inferior parietal & $-0.60(0.23)$ & $-0.48(0.4 \mathrm{I})$ & $-0.45(0.27)$ & 0.476 & 0.788 \\
\hline Inferior temporal & $-1.07(0.46)$ & $-0.32(0.47)$ & $-1.78(0.40)$ & 3.303 & 0.192 \\
\hline Isthmus cingulate & $1.52(0.66)$ & $1.81(1.10)$ & $1.17(0.76)$ & 0.084 & 0.959 \\
\hline Lateral occipital & $-1.77(0.5 \mathrm{I})$ & $-2.08(0.70)$ & $-1.88(0.38)$ & 0.176 & 0.916 \\
\hline Lateral orbitofrontal & $1.95(0.47)$ & $2.32(0.69)$ & 1.51 (0.39) & 2.132 & 0.344 \\
\hline Lingual & $-0.53(0.37)$ & $-0.75(0.55)$ & $-0.7 \mid(0.38)$ & 0.036 & 0.982 \\
\hline Medial orbitofrontal & $3.16(0.53)$ & $2.54(0.60)$ & $0.61(0.48)$ & 12.245 & $0.002 *$ \\
\hline Middle temporal & $-0.49(0.38)$ & $-0.98(0.63)$ & $-0.05(0.49)$ & 0.276 & $0.87 I$ \\
\hline Parahippocampal & $0.73(0.59)$ & $0.05(1.14)$ & $-0.14(0.84)$ & 0.263 & 0.877 \\
\hline Paracentral & $0.34(0.54)$ & $-0.66(0.58)$ & $1.53(0.43)$ & 7.269 & $0.026 *$ \\
\hline Pars opercularis & $-0.70(0.5 \mathrm{I})$ & $-0.36(0.97)$ & $-0.35(0.39)$ & 0.278 & 0.870 \\
\hline Pars orbitalis & $0.82(0.70)$ & $-1.07(1.27)$ & $1.75(0.75)$ & 5.174 & 0.075 \\
\hline Pars triangularis & $-0.06(0.50)$ & $-1.22(0.94)$ & $1.19(0.47)$ & 6.510 & $0.039 *$ \\
\hline Pericalcarine & $-0.58(0.73)$ & $0.93(0.85)$ & $-1.35(0.73)$ & 2.768 & 0.251 \\
\hline Postcentral & $0.32(0.62)$ & $0.81(0.87)$ & $-0.01(0.38)$ & 1.890 & 0.389 \\
\hline Posterior cingulate & $3.10(0.6 \mathrm{I})$ & $1.82(0.72)$ & $3.78(0.54)$ & 4.365 & 0.113 \\
\hline Precentral & $0.86(0.46)$ & $0.84(0.43)$ & $0.63(0.39)$ & 1.349 & 0.509 \\
\hline Precuneus & $1.08(0.36)$ & $0.12(0.43)$ & $0.71(0.39)$ & 3.128 & 0.209 \\
\hline Rostral anterior cingulate & $2.96(1.11)$ & $2.70(0.90)$ & $2.80(0.73)$ & 0.075 & 0.963 \\
\hline Rostral middle frontal & $\mathrm{I} .52(0.4 \mathrm{I})$ & $0.84(0.43)$ & I.II (0.27) & 1.250 & 0.535 \\
\hline Superior frontal & $2.09(0.33)$ & $1.51(0.44)$ & $1.78(0.26)$ & 3.467 & 0.177 \\
\hline Superior parietal & $-0.77(0.47)$ & $0.51(0.68)$ & $0.18(0.26)$ & 5.011 & 0.082 \\
\hline Superior temporal & $-0.63(0.40)$ & $-0.77(0.57)$ & $0.08(0.39)$ & $5.54 I$ & 0.063 \\
\hline Supramarginal & $-0.20(0.53)$ & $-0.59(0.50)$ & $0.11(0.42)$ & 0.478 & 0.788 \\
\hline Frontal pole & $2.57(0.95)$ & $1.87(1.83)$ & $0.87(0.87)$ & 1.175 & 0.556 \\
\hline Temporal pole & $-2.30(1.08)$ & $-0.25(1.37)$ & $-0.88(0.63)$ & 1.402 & 0.496 \\
\hline Transverse temporal & $0.13(1.10)$ & $-0.09(0.86)$ & $-0.34(0.78)$ & 0.262 & 0.877 \\
\hline Insula & $0.12(0.40)$ & $-1.10(0.63)$ & $0.36(0.37)$ & 4.443 & 0.108 \\
\hline
\end{tabular}

Notes: Continuous variables were expressed as mean (SEM). *Significant at $P \leq 0.05$.

Abbreviations: Al, asymmetry index; ADHD, attention-deficit/hyperactivity disorder; TD, typically developing; ADHD-I, inattentive attention-deficit/hyperactivity disorder; ADHD-C, combined attention-deficit/hyperactivity disorder.

Table 4 Pairwise Comparisons of the Cortical Thickness Als Between the Two Subtypes in ADHD and TD Groups

\begin{tabular}{|l|l|l|l|}
\hline Cortical regions $(\mathbf{m m})$ & ADHD-I vs TD & ADHD-C vs TD & ADHD-C vs ADHD-I \\
\hline & adjusted $\boldsymbol{P}$ & adjusted $\boldsymbol{P}$ & adjusted $\boldsymbol{P}$ \\
Medial orbitofrontal & $0.003^{*}$ & 0.119 & 1.000 \\
Paracentral & 0.359 & $0.029 *$ & 0.659 \\
Pars triangularis & 0.238 & 0.062 & 1.000 \\
\hline
\end{tabular}

Notes: Bonferroni correction was used in pairwise comparisons. *Significant at $P \leq 0.05$.

Abbreviations: Al, asymmetry index; ADHD, attention-deficit/hyperactivity disorder; TD, typically developing; ADHD-I, inattentive attention-deficit/hyperactivity disorder; ADHD-C, combined attention-deficit/hyperactivity disorder. 
Table 5 The Cortical Gray-Matter Volume and SN Volume Als Between the Two Subtypes in ADHD and TD Groups

\begin{tabular}{|c|c|c|c|c|c|}
\hline \multirow{3}{*}{$\begin{array}{l}\text { Region } \\
\text { Cortical regions }\left(\mathrm{mm}^{3}\right)\end{array}$} & \multicolumn{3}{|l|}{ Al } & \multirow[t]{3}{*}{$\chi^{2}$} & \multirow[t]{2}{*}{$P$} \\
\hline & \multicolumn{2}{|l|}{ ADHD } & \multirow[t]{2}{*}{ TD } & & \\
\hline & ADHD-I & ADHD-C & & & \\
\hline Bankssts & $4.36(2.23)$ & $0.43(3.05)$ & $0.38(1.77)$ & 1.322 & 0.516 \\
\hline Caudal anterior cingulate & $-9.35(2.69)$ & $4.42(4.70)$ & $-7.61(2.60)$ & 6.390 & $0.04 I^{*}$ \\
\hline Caudal middle frontal & $6.02(2.15)$ & $6.72(2.59)$ & $7.68(1.70)$ & 0.137 & 0.934 \\
\hline Cuneus & $-4.74(\mathrm{I} .4 \mathrm{I})$ & $-6.68(2.30)$ & $-2.35(1.86)$ & 1.783 & 0.410 \\
\hline Entorhinal & $2.26(2.49)$ & $10.13(2.48)$ & $3.22(2.12)$ & 4.760 & 0.093 \\
\hline Fusiform & $\mathrm{I} .75(\mathrm{I} .5 \mathrm{I})$ & $0.38(1.34)$ & $1.52(1.04)$ & 0.615 & 0.735 \\
\hline Inferior parietal & $-8.90(1.19)$ & $-9.15(1.27)$ & $-9.75(0.95)$ & 0.213 & 0.899 \\
\hline Inferior temporal & $-0.9 \mathrm{I}(\mathrm{I} .5 \mathrm{I})$ & $2.4 \mathrm{I}(2.34)$ & $0.96(1.23)$ & 2.777 & 0.249 \\
\hline Isthmus cingulate & $3.71(1.31)$ & $9.97(1.56)$ & $3.86(1.29)$ & 7.616 & $0.022 *$ \\
\hline Lateral occipital & $-1.53(1.12)$ & $-2.24(2.11)$ & $-0.05(0.99)$ & I.77I & 0.412 \\
\hline Lateral orbitofrontal & $2.87(0.84)$ & $1.51(1.18)$ & $1.93(0.69)$ & 0.501 & 0.779 \\
\hline Lingual & $-0.73(1.11)$ & $-1.76(0.83)$ & $-2.44(0.86)$ & 1.419 & 0.492 \\
\hline Medial orbitofrontal & $-0.12(1.55)$ & $-2.06(1.52)$ & $-1.59(1.02)$ & 0.352 & 0.839 \\
\hline Middle temporal & $-5.40(1.29)$ & $-4.30(1.88)$ & $-2.98(0.92)$ & 2.267 & 0.322 \\
\hline Parahippocampal & $1.46(1.28)$ & $3.37(2.10)$ & $2.50(1.54)$ & 1.044 & 0.593 \\
\hline Paracentral & $-5.22(2.03)$ & $-9.00(2.04)$ & $-3.79(1.43)$ & 3.874 & 0.144 \\
\hline Pars opercularis & $7.97(1.88)$ & $10.57(2.14)$ & $8.88(1.90)$ & 1.076 & 0.584 \\
\hline Pars orbitalis & $-11.49(1.70)$ & $-13.20(1.61)$ & $-8.66(1.25)$ & 4.126 & 0.127 \\
\hline Pars triangularis & $-7.15(1.75)$ & $-10.88(2.11)$ & $-7.99(1.59)$ & 1.616 & 0.446 \\
\hline Pericalcarine & $-4.73(1.17)$ & $-8.45(1.25)$ & $-7.48(1.5 \mathrm{I})$ & 5.137 & 0.077 \\
\hline Postcentral & $1.66(1.47)$ & $2.86(I .4 I)$ & 3.65 (1.27) & 1.438 & 0.487 \\
\hline Posterior cingulate & $2.00(2.17)$ & $5.51(2.30)$ & $0.64(1.42)$ & 4.066 & 0.131 \\
\hline Precentral & $0.4 \mathrm{I}(\mathrm{I} . \mathrm{II})$ & $1.61(1.38)$ & $-0.26(0.69)$ & 2.099 & 0.350 \\
\hline Precuneus & $-0.28(1.25)$ & $-1.88(1.31)$ & $-0.43(0.98)$ & I.I87 & 0.552 \\
\hline Rostral anterior cingulate & $9.91(2.39)$ & $15.38(4.77)$ & $|2.3|(2.27)$ & 1.197 & 0.550 \\
\hline Rostral middle frontal & $2.22(1.30)$ & $-1.77(0.87)$ & $-1.22(0.64)$ & 3.079 & 0.215 \\
\hline Superior frontal & $3.61(0.89)$ & $0.39(0.91)$ & $2.32(0.59)$ & 6.000 & $0.050 *$ \\
\hline Superior parietal & $-0.08(1.31)$ & $1.86(1.67)$ & $0.63(0.83)$ & 2.465 & 0.292 \\
\hline Superior temporal & $1.90(1.03)$ & $2.36(1.20)$ & $3.09(0.79)$ & 0.231 & 0.891 \\
\hline Supramarginal & 5.37 (1.39) & $3.31(1.84)$ & $4.16(1.21)$ & 1.113 & 0.573 \\
\hline Frontal pole & $-11.72(2.18)$ & $-15.39(3.50)$ & $-14.05(1.46)$ & 2.413 & 0.299 \\
\hline Temporal pole & $3.86(1.84)$ & $7.86(2.56)$ & $4.32(1.33)$ & 1.326 & 0.515 \\
\hline Transverse temporal & $14.76(1.45)$ & 18.00 & $14.93(1.30)$ & 1.913 & 0.384 \\
\hline Insula & $-1.32(0.98)$ & $-0.61(0.54)$ & $-0.28(0.8 \mathrm{I})$ & 0.753 & 0.686 \\
\hline \multicolumn{6}{|l|}{ SN $\left(\mathrm{mm}^{3}\right)$} \\
\hline Thalamus & $-1.03(0.37)$ & $-2.08(0.53)$ & $-1.86(0.34)$ & 3.537 & 0.171 \\
\hline Caudate & $-1.89(0.53)$ & $-2.20(0.67)$ & $-1.52(0.47)$ & 1.076 & 0.584 \\
\hline Putamen & $1.50(0.48)$ & $2.29(0.70)$ & $1.68(0.29)$ & 1.212 & 0.546 \\
\hline Pallidum & $3.92(0.72)$ & $6.00(0.61)$ & $5.12(0.62)$ & 3.623 & 0.163 \\
\hline Hippocampus & $-1.66(0.59)$ & $-2.45(0.92)$ & $-0.96(0.45)$ & 2.062 & 0.357 \\
\hline Amygdala & $0.20(1.07)$ & $0.42(1.19)$ & $1.09(0.8 \mathrm{I})$ & 0.487 & 0.784 \\
\hline Accumbens & $-3.28(1.27)$ & $-4.64(1.43)$ & $-2.70(1.08)$ & 1.224 & 0.542 \\
\hline
\end{tabular}

Notes: Continuous variables were expressed as mean (SEM). *Significant at $P \leq 0.05$.

Abbreviations: SN, subcortical nucleus; Al, asymmetry index; ADHD, attention-deficit/hyperactivity disorder; TD, typically developing; ADHD-I, inattentive attentiondeficit/hyperactivity disorder; ADHD-C, combined attention-deficit/hyperactivity disorder.

AI of the isthmus cingulate was significantly different (adjusted $P=0.032$ ). There were no significant differences in the asymmetry of cortical gray-matter volume in the caudal anterior cingulate, isthmus cingulate, or superior frontal regions between ADHD-I and TD groups (adjusted $P>$ 0.05 for all) (Table 6). 
Table 6 Pairwise Comparisons of the Cortical Gray-Matter Volume Als Between the Two Subtypes in ADHD and TD Groups

\begin{tabular}{|l|l|l|l|}
\hline Cortical regions $\left(\mathbf{m m}^{\mathbf{3}}\right)$ & ADHD-I vs TD & ADHD-C vs TD & ADHD-C vs ADHD-I \\
\hline & adjusted $\mathbf{P}$ & adjusted $P$ & adjusted $\boldsymbol{P}$ \\
Caudal anterior cingulate & 1.000 & 0.076 & $0.050^{*}$ \\
Isthmus cingulate & 1.000 & $0.032^{*}$ & $0.036^{*}$ \\
Superior frontal & 0.520 & 0.407 & $0.045^{*}$ \\
\hline
\end{tabular}

Notes: Bonferroni correction was used in pairwise comparisons. *Significant at $P \leq 0.05$

Abbreviations: Al, asymmetry index; ADHD, attention-deficit/hyperactivity disorder; TD, typically developing; ADHD-I, inattentive attention-deficit/hyperactivity disorder; ADHD-C, combined attention-deficit/hyperactivity disorder.

\section{Correlation Between the Als of Cortical Regions and the Severity of ADHD Symptoms}

The cortical thickness AI of the medial orbitofrontal region was significantly positively correlated with ADHD symptoms (inattention) $(\mathrm{r}=0.394, P=0.002)$ (Figure 1). Even so, a significantly negative correlation was revealed between the cortical thickness AI of the paracentral and ADHD symptoms (inattention) $(\mathrm{r}=-0.296, P=0.019)$ (Figure 2). Besides, the increased severity of ADHD symptoms on the hyperactivity/impulsivity subscale was associated with a significantly increased cortical gray-matter volume $\mathrm{AI}$ in the isthmus cingulate $(\mathrm{r}=0.283, P=$ 0.026) (Figure 3).

\section{Discussion}

We investigated the changes in the asymmetry of cortical and subcortical structures between children and adolescents with ADHD and TD individuals. Moreover, this study was the first to explore differences in cortical and subcortical asymmetry between different subtypes of ADHD and TD participants. Although asymmetry in the SN in ADHD group did not reveal

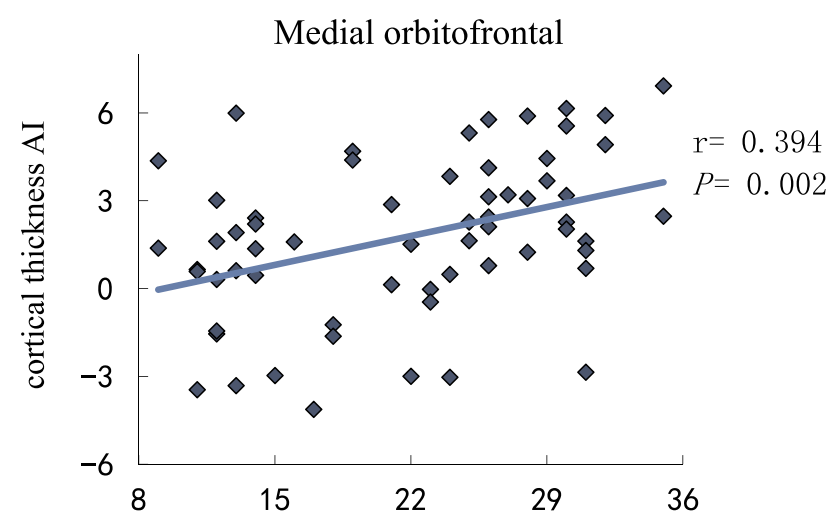

Inattention subscale score

Figure I Correlation between the cortical thickness Al in the medial orbitofrontal and the severity of ADHD symptoms on the inattention subscale score. significant differences (as we hypothesized), specific cortical regions showed differences in different subtypes of ADHD. In the present study, compared with TD individuals, different subtypes of ADHD demonstrated altered cortical asymmetry in the medial orbitofrontal, paracentral, pars triangularis, caudal anterior cingulate, isthmus cingulate, and superior frontal

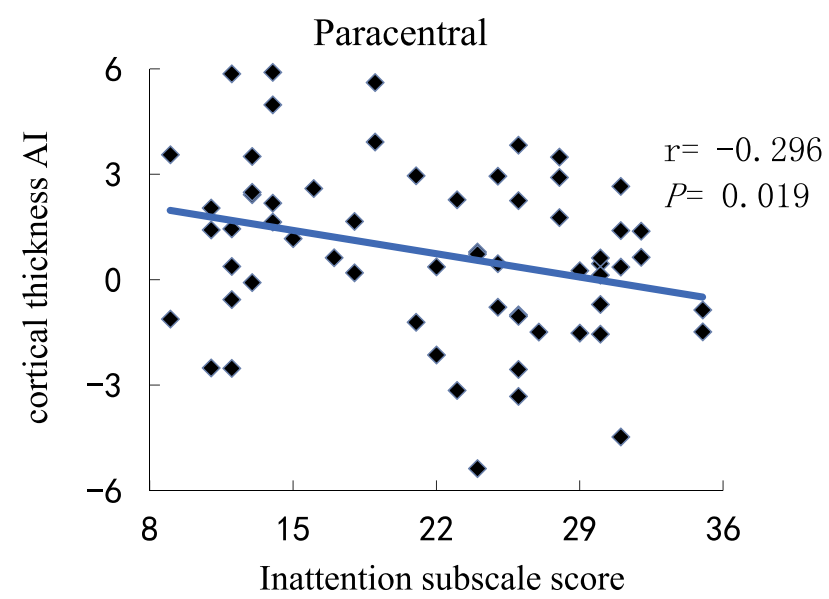

Figure 2 Correlation between the cortical thickness Al in the paracentral and the severity of ADHD symptoms on the inattention subscale score.

Isthmus cingulate

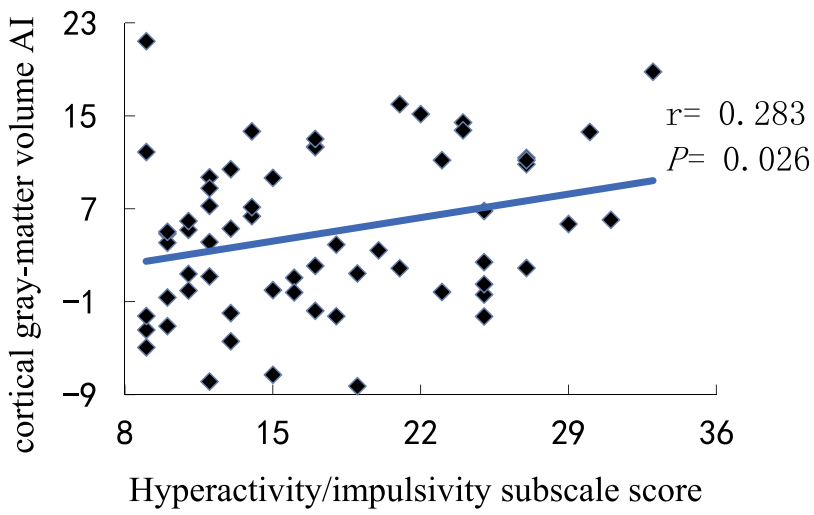

Figure 3 Correlation between the cortical gray-matter volume Al in the isthmus cingulate and the severity of ADHD symptoms on the hyperactivity/impulsivity subscale score. 
regions. These regions are parts of the orbitofrontal and cingulate circuits. Patients with impaired orbitofrontal and cingulate circuits can reveal automatic deficits and personality abnormalities, including behavioral disinhibition, emotional instability, and reduced motivation. ${ }^{29,30}$ These localized structural changes provide strong evidence that ADHD might have multiple deficits in the neural networks in the brain. The specific mechanism of cortical structural alterations is incompletely understood. A cadaver study by Chaudhry and coworkers supported the possibility that a reduced thickness in the pyramidal layer in the frontal lobe could elicit decreased cortex thickness. ${ }^{20}$ Pyramidal neurons are important components of the cortex, and their functions are closely related to advanced cognitive functions. Any alteration in these neurons might cause abnormal connections between and within the gyrus, leading to the clinical symptoms of ADHD.

The fronto-cortical and fronto-subcortical circuits are associated with controlled and automatic deficits. ${ }^{30,31}$ These functions could be impaired in children and adolescents with ADHD. The orbitofrontal cortex is connected to the frontal control system (eg, dorsolateral prefrontal cortex) and the limbic system (eg, cingulate and nucleus accumbens). ${ }^{32,33}$ The right medial orbitofrontal cortical gray-matter volume was increased in treatment-naive ADHD, whereas the cortical gray-matter volume comprises the cortical surface area and cortical thickness. ${ }^{24}$ An increased cortical gray-matter volume is accompanied by thinning of cortical thickness. These phenomena were consistent with the asymmetry in the cortical thickness of the orbitofrontal cortex measured between ADHD-I and TD groups in our study. Furthermore, a recent study showed that the ADHD-I group is slower than the TD group in terms of automatic processing speed. ${ }^{2}$ Combined with our results, the cortical abnormalities of the orbitofrontal cortex in the ADHD-I group might be related to automatic deficits. The structural abnormality in the orbitofrontal cortex might be a pathophysiological basis for the connectivity of the cortical-limbic system in ADHD. The paracentral lobule is located on the medial side of the frontal-parietal lobe and manages movement and sensation. In our study, the cortical asymmetry in the paracentral region was significantly different between ADHD-C and TD groups. Moreover, an increased inattention symptom score was correlated with decreased cortical thickness AI. A study by Zou and colleagues based on the variability of the restingstate fMRI signal showed increased right-lateralization in the paracentral lobule of ADHD patients, whereas there was no difference between the ADHD-C and ADHD-I groups in this region. $^{34}$
Impulsive aggression is observed widely in children with ADHD, and is linked to the unsatisfied expectation of a reward. ${ }^{33}$ Studies have shown that impulsive aggression is characterized by severe structural changes in the amygdala, anterior cingulate cortex, and orbitofrontal cortex. ${ }^{33,35-37}$ As we have shown, the asymmetry in the cortical graymatter volume in the isthmus cingulate was correlated with the hyperactivity/impulsivity score. However, a multivariate-analysis study demonstrated that the white-matter fibers of connectivity in the fronto-accumbal circuit and cortical thickness within the orbitofrontal cortex mainly explained aggression, but not impulsivity, in treatmentnaive children with ADHD. ${ }^{33}$ Those results were partially in accordance with our findings, and revealed that the cortical-thickness asymmetry in the medial orbitofrontal was associated with the inattention symptom score, but not the hyperactivity/impulsivity symptom score. The isthmus cingulate connects the cingulate gyrus to the parahippocampal gyrus (the function of which is controversial and might be related to verbal ability and adaptability) and pars triangularis which, as a part of Broca's area, plays a vital part in the processing of language and interpersonal information. ${ }^{38}$ In our study, participants with ADHD had asymmetry abnormalities in these two cortical regions compared with those in TD individuals. These abnormalities in the asymmetry of cortical structures might be a specific cue for verbal and interpersonal processing in children and adolescents with ADHD. The superior frontal region is primarily responsible for self-awareness, rational decision-making, and motor function. Our study demonstrated that ADHD had corticalvolume asymmetry in this region. A graph-theory approach has revealed that the dorsal superior frontal gyrus, central sulcus, and putamen in adults with ADHD have reduced nodal efficiency asymmetry. ${ }^{39}$ Also, we found alterations in cortical-volume asymmetry in the caudal anterior cingulate, isthmus cingulate, and superior frontal regions between ADHD-I and ADHD-C groups. The difference between the two subtypes of ADHD patients is that ADHD-C patients have mixed hyperactivity/impulsivity symptoms. Our results suggest that the asymmetry of cortical graymatter volume in the frontal and cingulate cortex might be the specific alterations between individuals with ADHD-I and ADHD-C subtypes.

\section{Limitations}

Our study had three main limitations. First, the study cohort was very small, so this study could be considered to be a pilot study. We must interpret with caution any 
asymmetry abnormalities that we distinguished. More participants are needed to obtain more robust results. Second, we did not have a patient cohort with the ADHD-HI subtype, and the results would have been more comprehensive if we had included all subtypes of ADHD. Third, we did not exclude ADHD patients taking medications, and some ADHD patients might have had a history of taking medications, which may have effects on brain structure (and our results).

\section{Conclusion}

Altered cortical and subcortical morphological asymmetry in children and adolescents with ADHD were predominantly located in cortical orbitofrontal and cingulate circuits. There were differences between patients with ADHD-I and ADHD-C subtypes in terms of the asymmetry of cortical regions in the cingulate and frontal cortex. These abnormalities were associated (at least in part) with the severity of ADHD symptoms. Hence, brain asymmetry could be an appropriate precursor of morphological alterations in neurodevelopmental disorders. These findings may provide potential monitoring for the valid diagnosis and treatment of ADHD.

\section{Acknowledgments}

This study was supported by The National Key Research and Development Program of China (2016YPC1306100) and the Special Fund of the Pediatric Medical Coordinated Development Center of Beijing Hospitals Authority (XTYB201812).

\section{Disclosure}

The authors report no conflicts of interest in this work.

\section{References}

1. Martino G. Automatic Deficits can lead to executive deficits in ADHD Mediterranean J Clin Psychol. 2017;5.

2. Capri T, Santoddi E, Fabio RA. Multi-source interference task paradigm to enhance automatic and controlled processes in ADHD. Res Dev Disabil. 2020;97:103542. doi:10.1016/j.ridd.2019.103542

3. Posner J, Polanczyk GV, Sonuga-Barke E. Attention-deficit hyperactivity disorder. Lancet. 2020;395(10222):450-462. doi:10.1016/S01406736(19)33004-1

4. Gallo EF, Posner J. Moving towards causality in attention-deficit hyperactivity disorder: overview of neural and genetic mechanisms. Lancet Psychiatry. 2016;3(6):555-567. doi:10.1016/S2215-0366(16) 00096-1

5. Willcutt EG. The prevalence of DSM-IV attention-deficit/hyperactivity disorder: a meta-analytic review. Neurotherapeutics. 2012;9(3):490499. doi:10.1007/s13311-012-0135-8
6. Faraone SV, Asherson P, Banaschewski T, et al. Attention-deficit/ hyperactivity disorder. Nature Reviews Disease Primers. 2015;1:1. doi:10.1038/nrdp.2015.20

7. Kong X-Z, Mathias SR, Guadalupe T, et al. Mapping cortical brain asymmetry in 17,141 healthy individuals worldwide via the ENIGMA Consortium. Proc Nat Acad Sci. 2018;115(22):E5154E5163. doi:10.1073/pnas.1718418115

8. Toga AW, Thompson PM. Mapping brain asymmetry. Nat Rev Neurosci. 2003;4(1):37-48. doi:10.1038/nrn1009

9. Takao H, Hayashi N, Ohtomo K. White matter asymmetry in healthy individuals: a diffusion tensor imaging study using tract-based spatial statistics. Neuroscience. 2011;193:291-299. doi:10.1016/j.neuroscience.2011.07.041

10. Okada N, Fukunaga M, Yamashita F, et al. Abnormal asymmetries in subcortical brain volume in schizophrenia. Mol Psychiatry. 2016;21 (10):1460-1466. doi:10.1038/mp.2015.209

11. Zuo Z, Ran S, Wang Y, et al. Asymmetry in cortical thickness and subcortical volume in treatment-naive major depressive disorder. Neuroimage Clin. 2019;21:101614. doi:10.1016/j.nicl.2018.101614

12. Sarica A, Vasta R, Novellino F, et al. MRI asymmetry index of hippocampal subfields increases through the continuum from the mild cognitive impairment to the Alzheimer's Disease. Front Neurosci. 2018;12:576. doi:10.3389/fnins.2018.00576

13. Woolard AA, Heckers S. Anatomical and functional correlates of human hippocampal volume asymmetry. Psychiatry Res. 2012;201 (1):48-53. doi:10.1016/j.pscychresns.2011.07.016

14. Ellison-Wright I, Ellison-Wright Z, Bullmore E. Structural brain change in attention deficit hyperactivity disorder identified by meta-analysis. BMC Psychiatry. 2008;8(1):51. doi:10.1186/1471-244X-8-51

15. Hoogman M, Bralten J, Hibar DP, et al. Subcortical brain volume differences in participants with attention deficit hyperactivity disorder in children and adults: a cross-sectional mega-analysis. Lancet Psychiatry. 2017;4(4):310-319. doi:10.1016/S2215-0366(17)30049-4

16. Berquin PC, Giedd JN, Jacobsen LK, et al. Cerebellum in attentiondeficit hyperactivity disorder. Morphometric MRI Study. 1998;50 (4):1087-1093.

17. Teicher MH, Anderson CM, Polcari A, et al. Functional deficits in basal ganglia of children with attention-deficit/hyperactivity disorder shown with functional magnetic resonance imaging relaxometry. Nat Med. 2000;6(4):470-473. doi:10.1038/74737

18. Parnaudeau S, O'Neill P-K, Scott, et al. Inhibition of mediodorsal thalamus disrupts thalamofrontal connectivity and cognition. Neuron. 2013;77(6):1151-1162. doi:10.1016/j.neuron.2013.01.038

19. Silk TJ, Vilgis V, Adamson C, et al. Abnormal asymmetry in frontostriatal white matter in children with attention deficit hyperactivity disorder. Brain Imaging Behav. 2016;10(4):1080-1089. doi:10.1007/ s11682-015-9470-9

20. Chaudhry R, Williams, et al. Changes in cortical thickness in the frontal lobes in schizophrenia are a result of thinning of pyramidal cell layers. Int Clin Psychopharmacol. 2011;26:e126-e127. doi:10.1097/01.yic.0000405847.48572.27

21. Nakao T, Radua J, Rubia K, et al. Gray matter volume abnormalities in ADHD: voxel-based meta-analysis exploring the effects of age and stimulant medication. Am J Psychiatry. 2011;168(11):1154-1163. doi:10.1176/appi.ajp.2011.11020281

22. Frodl T, Skokauskas N. Meta-analysis of structural MRI studies in children and adults with attention deficit hyperactivity disorder indicates treatment effects. Acta Psychiatr Scand. 2012;125(2):114-126. doi:10.1111/j.1600-0447.2011.01786.x

23. Meyer M, Liem F, Hirsiger S, et al. Cortical surface area and cortical thickness demonstrate differential structural asymmetry in auditoryrelated areas of the human cortex. Cerebral Cortex. 2013;24:25412552. doi:10.1093/cercor/bht094

24. Douglas PK, Gutman B, Anderson A, et al. Hemispheric brain asymmetry differences in youths with attention-deficit/hyperactivity disorder. Neuroimage Clin. 2018;18:744-752. doi:10.1016/j. nicl.2018.02.020 
25. Consortium HD. The ADHD-200 consortium: a model to advance the translational potential of neuroimaging in clinical neuroscience. Front Syst Neurosci. 2012;6:62. doi:10.3389/fnsys.2012.00062

26. Kaufman J, Birmaher B, Brent D, et al. Schedule for affective disorders and schizophrenia for school-age children-present and lifetime version (K-SADS-PL): initial reliability and validity data. J Am Acad Child Adolesc Psychiatry. 1997;36(7):980-988. doi:10.1097/ 00004583-199707000-00021

27. Vander Steene G. Wechsler intelligence scale for children-revised. Nederlandstalige Uitgave Verantwoording. 1986.

28. Pappas D. ADHD rating scale-iv: checklists, norms, and clinical interpretation. J Psychoeduc Assess. 2006;24(2):172-178. doi:10.1177/0734282905285792

29. Tekin S, Cummings JL. Frontal-subcortical neuronal circuits and clinical neuropsychiatry: an update. J Psychosom Res. 2002;53 (2):647-654. doi:10.1016/S0022-3999(02)00428-2

30. Batty MJ, Liddle EB, Pitiot A, et al. Cortical gray matter in attention-deficit/hyperactivity disorder: a structural magnetic resonance imaging study. J Am Acad Child Adolesc Psychiatry. 2010;49 (3):229-238.

31. Cubillo A, Rubia K. Structural and functional brain imaging in adult attention-deficit/hyperactivity disorder. Expert Rev Neurother. 2010;10(4):603-620. doi:10.1586/ern.10.4

32. Elliott R, Dolan RJ, Frith CD. Dissociable functions in the medial and lateral orbitofrontal cortex: evidence from human neuroimaging studies. Cereb Cortex. 2000;10(3):308-317. doi:10.1093/cercor/ 10.3.308
33. Cha J, Fekete T, Siciliano F, et al. Neural correlates of aggression in medication-naive children with ADHD: multivariate analysis of morphometry and tractography. Neuropsychopharmacology. 2015;40 (7):1717-1725. doi:10.1038/npp.2015.18

34. Zou H, Yang J. Exploring the brain lateralization in ADHD based on variability of resting-state fMRI signal. $J$ Atten Disord. 2018;25:1087054718816170. doi:10.1177/1087054718816170

35. Puiu AA, Wudarczyk O, Goerlich KS, et al. Impulsive aggression and response inhibition in attention-deficit/hyperactivity disorder and disruptive behavioral disorders: findings from a systematic review. Neurosci Biobehav Rev. 2018;90:231-246. doi:10.1016/j. neubiorev.2018.04.016

36. Longarzo M, Cavaliere C, Alfano V, et al. Electroencephalographic and neuroimaging asymmetry correlation in patients with attentiondeficit hyperactivity disorder. Neural Plast. 2020;2020:4838291. doi:10.1155/2020/4838291

37. Rubia K. Cognitive neuroscience of attention deficit hyperactivity disorder (ADHD) and its clinical translation. Front Hum Neurosci. 2018;12:100. doi:10.3389/fnhum.2018.00100

38. Beauregard M, Paquette V, Lévesque J. Dysfunction in the neural circuitry of emotional self-regulation in major depressive disorder. Neuroreport. 2006;17(8):843-846. doi:10.1097/01.wnr.0000220132.3 2091.9f

39. Li D, Li T, Niu Y, et al. Reduced hemispheric asymmetry of brain anatomical networks in attention deficit hyperactivity disorder. Brain Imaging Behav. 2019;13(3):669-684. doi:10.1007/s11682-018-9881-5
Neuropsychiatric Disease and Treatment

\section{Publish your work in this journal}

Neuropsychiatric Disease and Treatment is an international, peerreviewed journal of clinical therapeutics and pharmacology focusing on concise rapid reporting of clinical or pre-clinical studies on a range of neuropsychiatric and neurological disorders. This journal is indexed on PubMed Central, the 'PsycINFO' database and CAS, and is the official journal of The International Neuropsychiatric Association (INA). The manuscript management system is completely online and includes a very quick and fair peer-review system, which is all easy to use. Visit http://www.dovepress.com/testimonials.php to read real quotes from published authors. 